\title{
Concepts in Imaging and Microscopy \\ Choosing Objective Lenses: The Importance of Numerical Aperture and Magnification in Digital Optical Microscopy
}

\author{
DAVID W. PISTON \\ Department of Molecular Physiology and Biophysics, Vanderbilt University, \\ Nashville, Tennessee 37232-0615
}

\begin{abstract}
Microscopic images are characterized by a number of microscope-specific parameters - numerical aperture (NA), magnification (M), and resolution (R) — and by parameters that also depend on the specimen - for example, contrast, signal-to-noise ratio, dynamic range, and integration time. In this article, issues associated with the microscope-specific parameters NA, M, and R are discussed with respect to both widefield and laser scanning confocal microscopies. Although most of the discussion points apply to optical microscopy in general, the main application considered is fluorescence microscopy.
\end{abstract}

\section{Introduction}

The objective lens is arguably the most important component of any light microscope (Keller, 1995). Advances in digital imaging have completely changed the way that optical microscopy is performed, and have also changed the relevant specifications for objective lenses. Although lens design, construction, and quality have improved to keep up with the requirements of modern light microscopy, the markings on the lenses remain as they have been for decades. On the objective lens shown in Figure

Received 13 February 1998; accepted 28 May 1998

E-mail: dave.piston@mcmail.vanderbilt.edu

This is the second in a series of articles entitled " Concepts in Imaging and Microscopy." This series is supported by the Opto-Precision Instruments Association (OPIA) and was introduced with an editorial in the April 1998 issue of this journal (Biol. Bull. 194: 99). The first article in the series was written by Dr. Kenneth R. Castleman and appeared in the same issue (Biol. Bull. 194: 100-107).
1, the word "FLUAR" describes the type of lens design; although all manufacturers use similar types of designs, the nomenclature varies from company to company. The next most notable feature on the objective lens is the magnification $(\mathrm{M})$, which in the illustration is $100 \times$. It is written in the largest font of all the specifications, yet as is discussed here, it is not the most important parameter. This distinction belongs to the numerical aperture (NA), which is written next to the magnification, but in a smaller font, and in this case is 1.30 . The immersion medium for this objective is also given. Below the magnification and numerical aperture, the tube length $(\infty)$ and the coverslip thickness $(0.17 \mathrm{~mm})$ are given. Currently all manufacturers are offering infinity-corrected optics (denoted by the $\infty$ symbol), and most lenses are optimally corrected for a number 1.5 coverslip, nominally $170-\mu \mathrm{m}$ thick. Both of these parameters are important, but the objective lens will still function adequately for many applications with other tube lengths and coverslip thicknesses. However, because the manufacturers perform chromatic corrections in different ways, multi-color experiments - for instance, co-localization of two different colored immunofluorescent probes - should be performed using only sets of optics that were designed to work together. This applies not only to mixing lenses of different manufacturers, but also to mixing older and newer lines of optics. The working distance of the objective (the depth into the sample to which the lens can focus before it runs into the sample) is also a very important parameter, especially for confocal microscopy in thick biological samples. Despite the critical nature of this spec- 


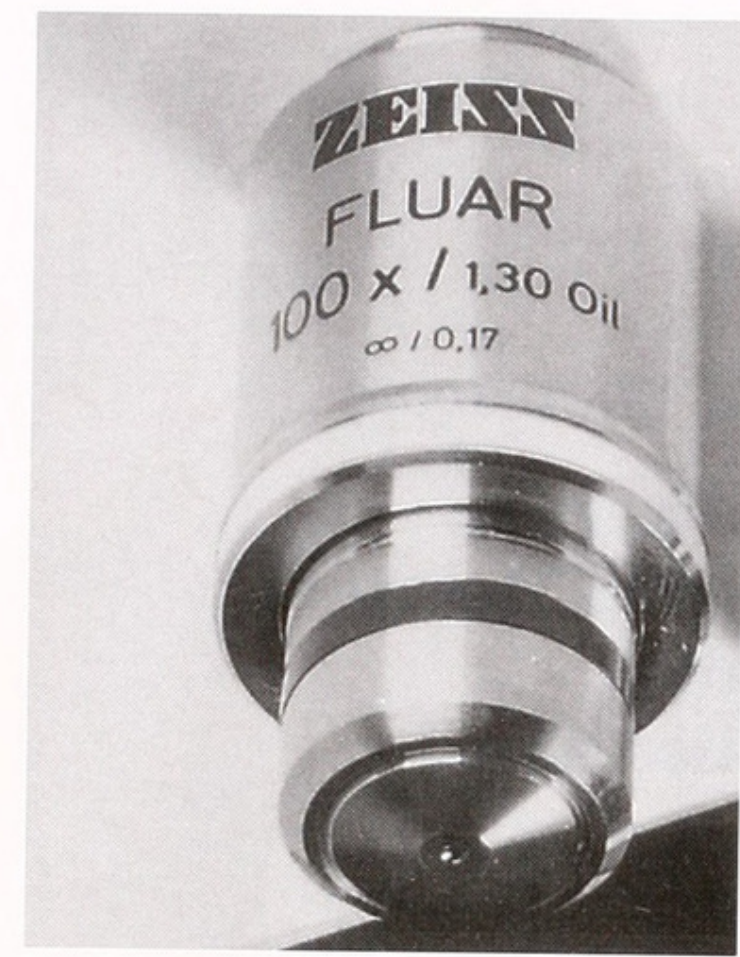

Figure 1. An objective lens with typical markings. Although this is a Zeiss lens, most manufacturers use similar markings.

ification, the working distance is not marked on most lenses. Nikon has now started writing the working distance on their CFI60 optics, and it is hoped that this trend will be followed by the other manufacturers.

This short article describes the relative importance of magnification and numerical aperture for digital optical microscopy. Traditionally, observations made with optical microscopes were detected by eye, and in this case, the size of the detector pixels - given by physiological factors in the human eye - is not optimal, so the magnification was increased so the sample could be "seen" better. In digital imaging, however, the magnification can be determined by the combination of resolution and detector pixel size. To understand the relative importance of NA and magnification, we must consider the basics of image formation and the effect of lens parameters on the resolution and information content in optical microscopy. Because the resolving power of an optical microscope is dependent only on the numerical aperture, magnification should be thought of as a secondary parameter whose optimal value can be determined by the NA, detector pixel size, and other instrumentindependent imaging parameters. Thus, NA is a more important parameter than magnification in digital imaging. The practical implications of this conclusion are described for two commonly used modes of fluorescence imaging: widefield epi-fluorescence microscopy with a CCD camera as the detector, and laser scanning confocal microscopy with photomultiplier tube detectors.

\section{Basics of Image Formation}

As might be guessed by looking at the markings on any objective lens, the magnification and numerical aper- ture are important for the image-formation properties of an optical microscope. Magnification for an optical instrument is defined as the relative enlargement of the image over the object. Although at first glance it would seem best to use the highest magnification possible, the maximal useful magnification is limited by the resolution of the imaging instrument (as described in the next paragraph). The definition of numerical aperture is more complicated. NA is defined by the half-angle of the objective's collection cone $(\alpha)$ and the index of refraction of the immersion medium $(\mathrm{n})$, and is expressed by $\mathrm{NA}=$ $\mathrm{n} \cdot \sin (\alpha)$ (Inoué and Spring, 1997, p. 32). The larger the cone of collected light, the higher the NA, and the more light that will be collected. Thus in practice, NA can be thought of as the amount of light that is collected by the objective lens; a high-NA lens collects more light than a low-NA lens. An analogy is with telescopes: a larger telescope collects more light just as a lens with a larger NA collects more light. Most optical microscopes also offer the option of secondary magnification between the objective lens and the detector. Use of such extra magnification may sometimes be required (see Table I), but should be avoided if possible since extra light loss is introduced.

As suggested above, resolution (as determined by the basic diffraction principles of light) limits the useful magnification in an optical microscope. Resolution $(\mathrm{R})$ is defined as the smallest distance that two objects can be apart and still be discerned as two separate objects. There are many mathematical definitions for resolution, but a simple and reasonable approximation is $\mathrm{R}=\lambda /(2 \cdot \mathrm{NA})$, where $\lambda$ is the wavelength of the light (Inoué and Spring, 1997, p. 31). This relationship indicates that when using a highNA lens and 500-nm (blue-green) light, the smallest resolvable distance is $\sim 200 \mathrm{~nm}$, or $0.2 \mu \mathrm{m}$, which agrees well with experimental values. One frequent point of confusion for microscope users is the difference between spatial resolution (the ability to distinguish multiple objects) and spatial precision (the ability to localize a single object). Many image-processing enhancements can be used to increase the precision of localization. For example, the path of a single microtubule can be determined to $\sim 10 \mathrm{~nm}$ precision by pixel-fitting (e.g., Ghosh and Webb, 1994) or deconvolution methods (e.g., Agard et al., 1989; Carrington et al., 1990; Holmes et al., 1995). However, this is not $10-\mathrm{nm}$ resolution; $10-\mathrm{nm}$ resolution means that two microtubules that are $10-\mathrm{nm}$ apart can be recognized as two separate tubules. If multiple objects are small and close together (that is, close enough that they cannot be resolved), then no amount of image processing can differentiate between several individual objects and a single object.

Since there is a minimum resolvable distance for every microscope, continuing to increase the magnification past a certain point will no longer increase the information 
content of the image. Further magnification beyond this point is sometimes referred to as "empty" or meaningless magnification. This is analogous to any digital image on a computer, where pixelation is observed when an image is magnified on the screen (this can be seen, for example, by repeatedly using the "zoom in" command in Adobe Photoshop). So the question obviously arises, how should the correct magnification be chosen? A good rule is to use the Nyquist criterion, which basically says that one should collect two points per resolution size (Inoué and Spring, 1997, p. 513). Collecting images in this manner maximizes the information content.

The use of $\lambda /(2 \cdot \mathrm{NA})$ for the resolution criterion, and of R/2 for the sampling rate are both arbitrary. Many microscopists select other resolution criteria, but all of these choices are only mathematical approximations of the same physical properties. Use of any other resolution criteria would not affect the arguments presented here, although the numbers (e.g., those shown in Table I) would change slightly.

Some attention should also be given to special considerations for fluorescence microscopy (Rost, 1992). Since fluorescence is subject to fluorophore saturation and photobleaching effects that do not affect other optical methods, the highest possible light collection efficiency is desirable. This consideration dictates that the highest possible NA should be used. However, the highest NA lenses $(\mathrm{NA}=1.40)$ are usually of a "plan-Apochromat" design; this type of lens consists of up to 14 elements and has a lower transmittance than a "Fluor" design. Also, if aqueous samples are used, the actual NA is limited to $\sim 1.2$ because of total internal reflection for higher collection angles at the interface between water and coverslip (Inoué and Spring, 1997, pp. 53-55). For these reasons, fluorescence from an aqueous sample appears brighter through a $100 \times / 1.30$ NA FLUAR (as shown in Fig. 1) than through a $100 \times / 1.40$ plan-Apochromat objective lens.

Finally, it should be noted that improvements in almost every aspect of lens design and construction (e.g., computer design of complex lens combination, automated grinding of arbitrary lens shapes, new optical materials for both lenses and coatings, and computer-controlled thin film deposition for precise optical coatings) make modern objective lenses superior to and more reliable than older lenses. Although many older lenses are superb, variables during their construction made finding a good one somewhat challenging, and many researchers just took what came. Today's lenses are consistently of high quality, and also offer higher transmission efficiency and lower autofluorescence than did older lenses.

Numerical aperture, magnification, and resolution in widefield microscopy

In a digital widefield (conventional fluorescence) microscope, the image is projected onto an imaging detector (usually a CCD camera) that takes the place of the eye. Thus, to optimize the information content of the resulting digital image, the pixels on the detector must be matched to the desired image resolution. As described above, the radius of a diffraction-limited spot, $\mathrm{R}_{\text {diff }} \approx N /(2 \mathrm{NA})$, is a good quantity to use for the definition of resolution. In the image plane, this spot will still give the smallest resolvable object, but the width of the spot will now be $\mathrm{M} \cdot \mathrm{R}$. Based on the Nyquist criterion, the desired sampling rate should be twice the resolution, so we want a pixel size in the object of $\mathrm{R}_{\text {samp }} \approx \mathrm{R}_{\mathrm{diff}} / 2$. In practice, the pixel size in widefield microscopy is fixed by the imaging camera used, so the magnification is the only variable that can be adjusted. For the purposes of these calculations, we can assume $\lambda=0.5 \mu \mathrm{m}$ (a good approximation for fluorescein (FITC) imaging). We can determine the optimal $\mathrm{M}$ to be used for a given pixel size by matching the sampling resolution in the image plane to the pixel size (P) by $\mathrm{P}=\mathrm{M} \cdot \mathrm{R}_{\text {samp }}$. Table $\mathrm{I}$ shows the results of this calculation for two typical pixel sizes: $24 \mu \mathrm{m}$ (an older SITe 512D CCD chip) and $6.8 \mu \mathrm{m}$ (the more modern Kodak KAF1400 CCD chip). As can be seen from the table, the older chips (with their larger pixel sizes) require higher magnification. For these larger pixels, an extra intermediate magnification of 2.5 would be required to maximize the information content of an image collected with a $100 \times / 1.3 \mathrm{NA}$ objective lens, and in fact cameras that use the older SITe chip usually have some extra magnification built into them. As micro-fabrication technology continues to advance, however, the need for high magnification lenses will decrease. Obviously, it is not possible to purchase a $72 \times / 1.30 \mathrm{NA}$ lens (although given the popularity of the KAF1400 CCD chip, perhaps it should be), so these optimal magnifications can only serve as a guide for selection of the best objective lens. Further calculations, such as those presented in the table, reveal that a camera with a pixel size of $5.4 \mu \mathrm{m}$ would be ideal for use with many existing lenses, such as $60 \times / 1.4 \mathrm{NA}$, $40 \times / 0.90 \mathrm{NA}$, and $25 \times / 0.60 \mathrm{NA}$. It should be noted, however, that as pixel sizes get smaller, the dynamic range of the detector may be reduced. For instance, the $5.4-\mu \mathrm{m}$ pixels would likely be filled by fewer than 20,000 counts, which would limit the detector to 14-bit dynamic range. This is in contrast to larger pixel sizes (i.e., the $24 \mu \mathrm{m}$ in the SITe 512D CCD chip), which can easily deliver $>16$-bit dynamic range. For applications that require high precision, such as deconvolution methods, smaller pixels may be unworkable.

Numerical aperture, magnification, and resolution in laser scanning microscopy

Much has been made of the improvement in resolution provided by confocal microscopy. But this improvement is at best minimal, and is only attained for extremely 
Table I

Optimal magnification for detectors with different pixel sizes calculated for five numerical apertures

\begin{tabular}{|c|c|c|c|c|c|}
\hline & \multicolumn{5}{|c|}{ Numerical aperture (NA) } \\
\hline & 1.40 & 1.30 & 0.90 & 0.60 & 0.30 \\
\hline \multicolumn{6}{|c|}{ Calculated resolutions $(\mu \mathrm{m})$} \\
\hline $\mathrm{R}_{\text {diff }}$ & 0.18 & 0.19 & 0.28 & 0.42 & 0.83 \\
\hline $\mathrm{R}_{\text {samp }}$ & 0.090 & 0.095 & 0.140 & 0.210 & 0.415 \\
\hline \multicolumn{6}{|l|}{ Magnification $(\times)$} \\
\hline $24-\mu \mathrm{m}$ pixels* & 267 & 253 & 171 & 114 & 58 \\
\hline $6.8-\mu \mathrm{m}$ pixels $\dagger$ & 76 & 72 & 49 & 32 & 16 \\
\hline
\end{tabular}

* Represents the SITe512D CCD chip.

$\doteqdot$ Represents the Kodak KAF1400 CCD chip.

small pinholes. In practical fluorescence microscopy, the pinhole must be opened somewhat to increase the efficiency of fluorescence collection. In fact, the pinhole is almost always opened enough to negate any resolution enhancement (Sandison et al., 1995). In this practical case, there is an improvement in rejection of out-of-focus background, but the resolution is still given by $R_{\text {diff }} \approx N$ (2 NA), so the ideal sampling resolution remains as shown in Table I.

A key point in laser scanning microscopy is that there is no longer a fixed pixel size. Because the field over which the laser is scanned can be varied (this variation is usually called "zoom," or more appropriately "electronic zoom," and is analogous to using an optical zoom lens), the sampling resolution can be easily changed. For this reason, users often have a lot of trouble choosing lenses when they switch to laser scanning confocal microscopy. For instance, a $40 \times$ lens with a zoom factor of 2.5 is basically equivalent to a $100 \times$ lens with no extra zoom. Thus, a $40 \times / 1.3 \mathrm{NA}$ lens should be chosen over an equivalent $100 \times / 1.3 \mathrm{NA}$ lens, because it offers a potentially larger field of view with no fall-off in light collection or resolution.

In laser scanning confocal microscopy, two other parameters must be considered. First is the size of the detector pinhole, which depends on the magnification. Most confocal microscopes have an adjustable pinhole that is easily set to match the magnification (e.g., for equivalence, a $60 \times$ lens needs a pinhole 1.5 -fold larger than a $40 \times$ lens). Secondly, the lens design for confocal microscopy may, in some cases, be more important than either $\mathrm{M}$ or NA. This is especially true for co-localization experiments, in which the chromatic corrections of a planApochromat make it preferable despite its lower lightcollection efficiency (the same trade-off must be considered for any three-dimensional microscopies based on widefield and deconvolution methods, as well). Regardless of the lens design, however, a lower magnification lens (of equivalent NA) is almost always preferable, because it offers a larger field-of-view, and delivers equivalent resolution.

\section{Acknowledgments}

This work was supported by an Arnold and Mabel Beckman Foundation Young Investigator Award, NIH grant DK53434, and the Vanderbilt Cell Imaging Resource (underwritten by CA68485 and DK20593).

\section{Literature Cited}

Agard, D. A., Y. Hiraoka, P. Shaw, and J. W. Sedat. 1989. Fluorescence microscopy in three dimensions. Methods Cell Biol. 30: $353-$ 377.

Carrington, W. A., K. E. Fogarty, and F. S. Fay. 1990. 3D fluorescence imaging of single cells using image restoration. Pp. 53-72 in Non-invasive Techniques in Cell Biology. J. K. Foskett and S. Grinstein, eds. Wiley-Liss, New York.

Ghosh, R. N., and W. W. Webb. 1994. Automated detection and tracking of individual and clustered cell surface low density lipoprotein receptor molecules. Biophys. J. 66: 1301-1318.

Holmes, T. J., S. Bhattacharyya, J. A. Cooper, D. Hanzel, V. Krishnamurthi, W. Lin, B. Roysam, D. H. Szarowski, and J. N. Turner. 1995. Light microscopic images reconstructed by maximum likelihood deconvolution. Pp. 389-402 in The Handbook of Biological Confocal Microscopy, 2nd Edition. J. Pawley, ed. Plenum, New York.

Inoué, S., and K. R. Spring. 1997. Video Microscopy: the Fundamentals, 2nd Edition. Plenum, New York.

Keller, H. E. 1995. Objective lenses for confocal microscopy. Pp. 111-126 in The Handbook of Biological Confocal Microscopy, 2nd Edition. J. Pawley, ed. Plenum, New York.

Rost, F. W. D. 1992. Fluorescence Microscopy. Cambridge University Press, Cambridge, UK.

Sandison, D. R., D. W. Piston, R. M. Williams, and W. W. Webb. 1995. Resolution, background rejection, and signal-to-noise in widefield and confocal microscopy. App. Optics 34: 3576-3588. 


\section{$2 \mathrm{BHL}$ Biodiversity Heritage Library}

Piston, D W. 1998. "Choosing Objective Lenses: The Importance of Numerical Aperture and Magnification in Digital Optical Microscopy." The Biological bulletin 195, 1-4. https://doi.org/10.2307/1542768.

View This Item Online: https://www.biodiversitylibrary.org/item/17298

DOI: https://doi.org/10.2307/1542768

Permalink: https://www.biodiversitylibrary.org/partpdf/25608

\section{Holding Institution}

MBLWHOI Library

\section{Sponsored by}

MBLWHOI Library

\section{Copyright \& Reuse}

Copyright Status: In copyright. Digitized with the permission of the rights holder.

License: http://creativecommons.org/licenses/by-nc-sa/3.0/

Rights: https://biodiversitylibrary.org/permissions

This document was created from content at the Biodiversity Heritage Library, the world's largest open access digital library for biodiversity literature and archives. Visit BHL at https://www.biodiversitylibrary.org. 